\title{
Correlation between TyG (Fasting Triglyceride- glucose) Index, TyG BMI Index \& Liver Transaminases in Women with Polycystic Ovary Disease Syndrome
}

\author{
Gayatri Chandrashekhar Gawade ${ }^{1}$, Geeta Shripad Wadadekar ${ }^{2}$, \\ Meghana Khandu Padwal ${ }^{3}$, Aman Pritish Aher ${ }^{4}$, \\ Tushar Madhav Panchnandikar ${ }^{5}$ \\ ${ }^{1}$ Assistant Professor, Department of Biochemistry, Bharati Vidyapeeth (Deemed to be) University Medical \\ College, Pune-43 \\ ${ }^{2}$ Assistant Professor, Department of Obstetrics and Gynecology, Bharati Vidyapeeth (Deemed to be) University \\ Medical College, Pune-43 \\ ${ }^{3}$ Professor and Head, Department of Biochemistry, Bharati Vidyapeeth (Deemed to be) University \\ Medical College, Pune-43 \\ ${ }^{4}$ Undergraduate Student, Bharati Vidyapeeth (Deemed to be) University Medical College, Pune-43 \\ ${ }^{5}$ Professor and Head, Department of Obstetrics and Gynecology, Bharati Vidyapeeth (Deemed to be) University \\ Medical College, Pune-43 \\ Corresponding Author: Geeta Shripad Wadadekar
}

\begin{abstract}
Background: Polycystic Ovary Disease Syndrome (PCOS) and NAFLD are interlinked with the common culprit i.e. Insulin resistance (IR). The triglyceride and glucose index (TyG) has been recommended as a reliable and simple surrogate index for IR. So we evaluated TyG index, TyG-BMI index as a marker of IR and its correlation with liver transaminases (ALT, AST) to detect NAFLD in women with PCOS.

Aim and Objectives: Correlation of TyG index and TyG-BMI index and liver transaminases (ALT, AST) in study groups.

Materials and methods: A prospective study was done on 25 participants (14 controls and 11 cases of PCOS according to Rotterdam's criteria). The TG, FBG, ALT and AST were analyzed on autoanlyzers. TyG -BMI index was calculated by the formula and correlation was done.

Result: The ROC Curve showed the optimal cut off value for TyG index as 4.55 and TyG-BMI index in PCOS as 104.76. The positive linear regression was 2.55 for TyG index indicating that test was associated with IR. TyG index has shown the better performance as compared to TyG BMI index. No significant correlation was
\end{abstract}

found between TyG index, TyG BMI index and transaminases.

Conclusion: TG and FBG are inexpensive and routinely performed investigations. TyG index which can be easily calculated makes it the first choice screening alternative to detect risk of IR in PCOS women.

Keywords: PCOS, TyG -BMI index, Nonalcoholic fatty liver disease, liver transaminases

\section{INTRODUCTION}

With global shift in disease patterns from communicable to non-communicable diseases, India is facing the challenge of surge in NCD over last 3 decades. ${ }^{(1)}$

Metabolic syndrome refers to the cooccurrence of several known cardiovascular risk factors, including insulin resistance, obesity, atherogenic dyslipidemia and hypertension. ${ }^{(2)}$

Polycystic ovary syndrome is a heterogeneous endocrine disorder that affects about one in 15 women worldwide (3), prevalence in India ranging from 3.7 to 22.5 percent. $^{(4)}$ 
PCOS is frequently accompanied by IR and obesity and is characterized by ovulatory dysfunction, hyperandrogenism and polycystic ovarian morphology. ${ }^{(5)}$

A spectrum of hepatic pathologies ranging from simple hepatic steatosis to steatohepatitis and cirrhosis due to nonalcoholic causes is characteristic of NAFLD (nonalcoholic fatty liver disease).

Insulin resistance induces an increased hepatic de novo lipogenesis. Ineffective suppression of adipose tissue lipolysis results in an excessive amount of circulating fatty acids that lead to the development of simple hepatic steatosis. The liver fat accumulation by triglyceride deposition occurs simultaneously with the increased hepatic free fatty acid flux. Toxic levels of lipid-derived metabolites result in mitochondrial dysfunction, endoplasmic reticulum stress, and activation of multiple inflammatory pathways High levels of circulating fatty acids may further aggravate hepatic insulin resistance through deleterious effects on insulin signalling pathways by activation of serine kinase, augmenting the vicious cycle. ${ }^{(6)}$

There is close association of NAFLD with obesity, dyslipidemia and type 2 diabetes mellitus, which also forecasts the uptrend of cardiovascular disease risk. ${ }^{(7)}$

The usual observed biochemical pattern in hepatic steatosis due to NAFLD is of increased levels of transaminases, with alanine aminotransferase (ALT) levels exceeding those of aspartate aminotransferase (AST). ${ }^{(8}$ ) Liver enzymes may be normal in up to $78 \%$ of patients with NAFLD. ${ }^{(9)}$

Data from clinical studies and metaanalyses indicate a higher prevalence of NAFLD in women with PCOS (34\% to $70 \%$ ) compared to (14\% to $34 \%$ ) healthy women. ${ }^{(10)}$

Why test correlation between IR \& transaminases (biochemical marker of NAFLD)?

Why in women with PCOS?
- Both PCOS \&NAFLD are pathophysiologically interlinked, insulin resistance being the common culprit.

- Both conditions indicate high risk for developing long term adverse health consequences.

- There is opportunity for early detection $\&$ intervention.

The gold standard for directly measuring insulin sensitivity in humans is the hyperinsulinemia euglycemic glucose clamp, but it is time consuming, labor intensive, expensive and technically demanding.

The homeostatic model assessment of IR (HOMA-IR) is another method of measurement of IR used in scientific studies as it is simple and noninvasive.

The product of TG and FPG calculated as triglyceride and glucose index (TyG) has been studied as a surrogate index for insulin resistance which is given by following formula. ${ }^{(11)}$

\section{TyG index $=\ln [$ FPG $(\mathrm{mg} / \mathrm{dL}) \times$ TG $(\mathrm{mg} / \mathrm{dL})] / 2$}

It has been reported that the TyG index has high sensitivity for recognizing insulin resistance in apparently healthy subjects, as compared to the HOMA-IR: homeostasis model assessment of insulin resistance index ${ }^{(12)}$

Because TyG index is calculated from triglyceride levels, this marker may also reflect the presence of fatty liver.

In a cohort of 50 asymptomatic women from Mexico, the association between the TyG index and liver steatosis has been demonstrated. ${ }^{(13)}$

So, we wanted to evaluate $\mathrm{TyG}$ index and TyG -BMI index (TyG -BMI index $=$ TyG index $\times$ BMI) ${ }^{(11)}$ as a marker of insulin resistance and study their association with liver transaminases (ALT, AST) in women with PCOS.

\section{MATERIAL AND METHODS}

A cross-sectional, prospective study was conducted at University Medical College Tertiary Care Hospital Pune, 
Maharashtra in the duration from Feb 2018 to July 2018 of 6 months.

The diagnosed cases of PCOS with age more than $18 \mathrm{yrs}$ and according to the Rotterdam's criteria ${ }^{(14)}$, attending the Obstetrics and Gynecology OPD following the inclusion criteria were included in the study. Healthy age matched controls with no clinical and radiological evidence of PCOS were included in the study. Research protocol was submitted to \& approval was sought from institutional ethics committee. (BVDUMC/IEC/9) Informed consent was obtained from the participants. Inclusion criteria included women diagnosed with PCOS by Rotterdam criteria \& giving consent. Criteria included clinical or biochemical hyperandrogenism, olio- anovulation, polycystic morphology on ultrasound scan and exclusion of related disorders. Exclusion criteria included known diabetes, hypothyroidism, liver, kidney, or heart failure, neoplastic or any medical problem, on steroid hormones or OC Pills in last 3 months on drugs known to have effects on lipid metabolism during the past 2 years, not willing to participate.

Detailed clinical examination of the participants was done. The anthropometric parameters like weight in $\mathrm{kg} \&$ height in meters were measured, BMI ${ }^{(15)}$ calculated.

All the participants visiting the hospital and following the inclusion criteria during the study period were included in the study. Sample size was calculated by convenience sampling technique. Fig1

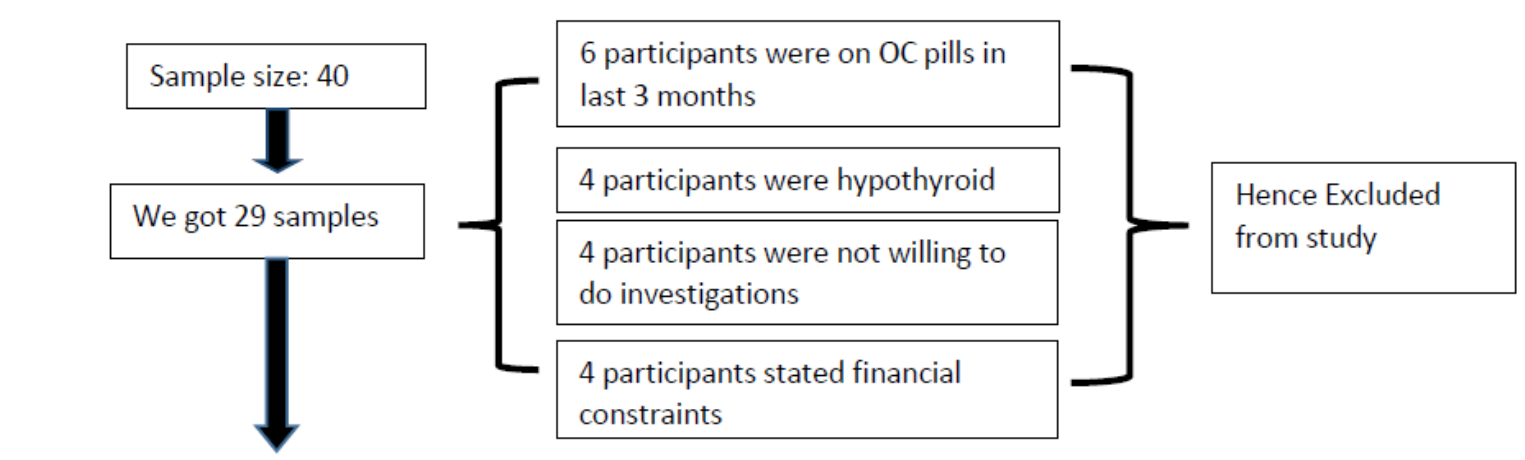

We got 11 cases which were included for statistical analysis

Fig.1.Flow chart showing the selection of cases.

\section{METHODOLOGY}

$4 \mathrm{ml}$ blood sample was collected from antecubital fossa without venous occlusion. Sample collection was done in Central Clinical Laboratory with all aseptic precautions after 12-14 hrs fasting. Blood was allowed to clot for one hour. Serum was separated after centrifugation at $2000 \mathrm{rpm}$ for 10 minutes. Separated serum samples were subjected for estimation of following parameters on Randox Biochemistry auto analyzer.

Table no 1: Table showing the methods used in estimation of parameters

\begin{tabular}{|c|c|l|}
\hline Sr.No. & Test & \multicolumn{1}{c|}{ Method } \\
\hline 1 & Glucose & Enzymatic Glucose Oxidase-Peroxidase \\
\hline 2 & Triglycerides & GPO/PAP \\
\hline 3 & ALT & IFCC without Pyridoxal Phosphate $37^{\circ} \mathrm{c}$ \\
\hline 4 & AST & IFCC without Pyridoxal Phosphate $37^{\circ} \mathrm{c}$ \\
\hline
\end{tabular}

Above facilities are available at central clinical laboratory of the hospital.

\section{Statistical analysis}

Scientific Package for Social Sciences (SPSS) version 19.0 was used for the analysis. Differences between more than two groups were estimated using one-way ANOVA test. Correlation coefficient was calculated to study the correlation between the study variables.

ROC curve analysis was performed to determine the area under curve and sensitivity and specificity.

Sensitivity and specificity of the TyG index and TyG BMI index were estimated as function of insulin resistance. 
The area under the ROC curve (AUC), as a summary of the overall diagnostic accuracy of the test, was estimated.

ROC curves that are shifted to the left with AUCs near 1 are considered as the best markers.

\section{RESULTS}

The average age was 27.5 years in the cases and 32.5 years in controls.

In cases, the mean BMI was $25.5 \pm$ $8.7 \mathrm{~kg} / \mathrm{m}^{2}$; with 7 pre obese $(27.42 \pm 3.9$ $\left.\mathrm{kg} / \mathrm{m}^{2}\right)$ and 4 nonobese $\left(22.57 \pm 3.6 \mathrm{~kg} / \mathrm{m}^{2}\right)$ patients. The mean BMI in controls was $25.66 \pm 7.3 \mathrm{~kg} / \mathrm{m}^{2}$; with 6 pre obese $(27.5 \pm$ $\left.3.1 \mathrm{~kg} / \mathrm{m}^{2}\right)$ and 8 nonobese $(22.0 \pm 1.7$ $\mathrm{kg} / \mathrm{m}^{2}$ ) women

Cases \& controls were grouped as obese or lean as per their BMI according to World Health Organization (WHO) criteria. (16) As shown in the figure 1. The Box and Whisker plot is showing the relation

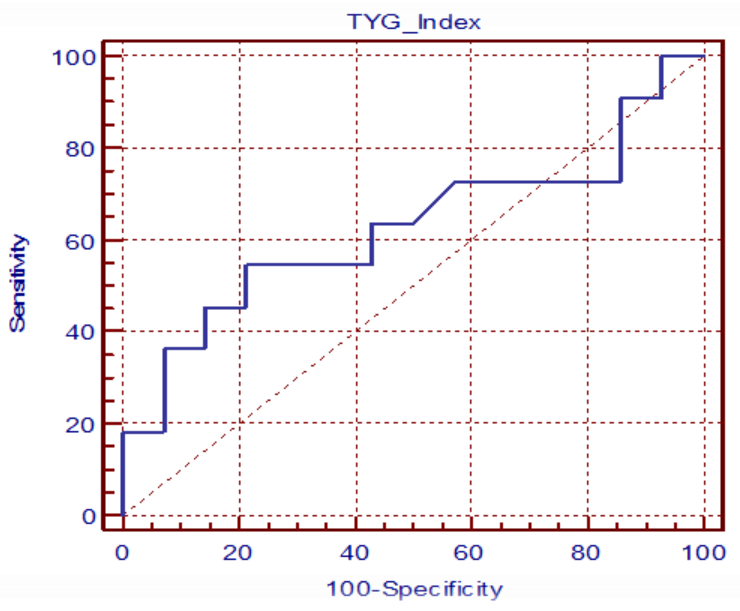

Fig. 3: ROC Curve for TyG Index between BMI, cases and controls. BMI was found to be correlated with TyG BMI index with correlation coefficient (0.77) and $\mathrm{p}$ value less than 0.05 ; findings are consistent with the study of R. Vidya Bharathi et al. ${ }^{(17)}$

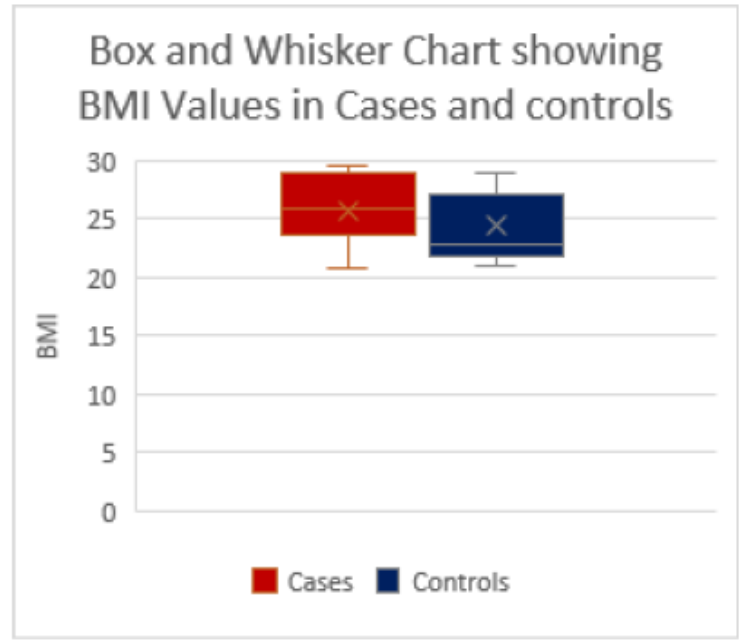

Fig. 2: Box and Whisker Chart showing BMI Values

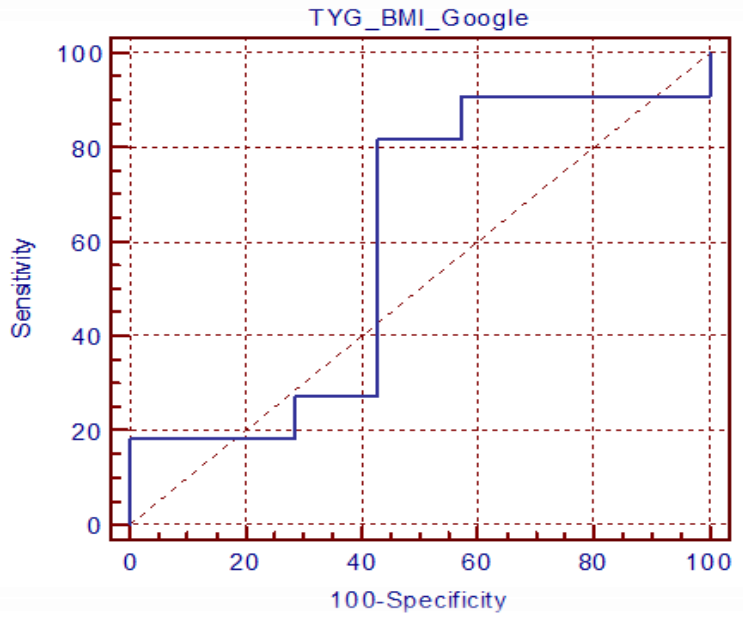

Fig.4: ROC Curve for TyG-BMI Index

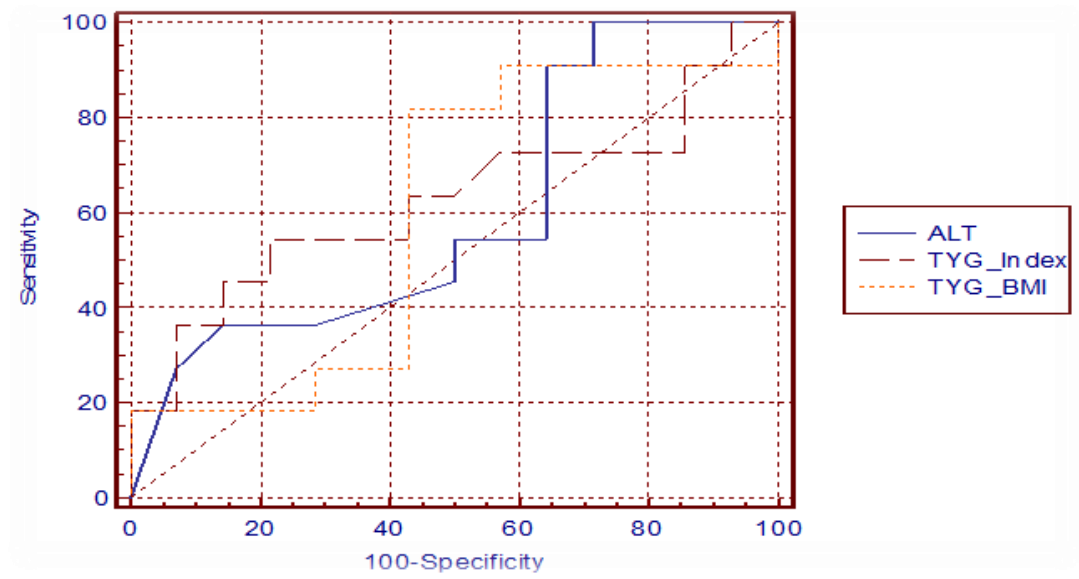

Fig. 5: ROC Curve for TyG Index, TyG BMI Index and AST /ALT 
Gayatri Chandrashekhar Gawade et.al. Correlation between TyG (fasting triglyceride-glucose) index, TyG BMI index \& liver transaminases in women with polycystic ovary disease syndrome.

Table 2: ROC Curve Analysis for TyG Index, TyG BMI Index and AST/ALT

\begin{tabular}{|c|c|c|c|c|c|c|c|}
\hline & $\begin{array}{c}\text { Area under the ROC } \\
\text { curve }\end{array}$ & $\begin{array}{c}\text { Standard } \\
\text { error }\end{array}$ & $\begin{array}{c}95 \% \text { Confidence } \\
\text { interval }\end{array}$ & Sensitivity & Specificity & $+\mathrm{LR}$ & -LR \\
\hline TyG Index & 0.627 & 0.113 & 0.412 to 0.810 & $\begin{array}{c}54.5 \\
(23.5-83.1) \\
\end{array}$ & $\begin{array}{c}78.6 \\
(49.2-95.1) \\
\end{array}$ & 2.55 & 0.58 \\
\hline $\begin{array}{l}\text { TyG BMI } \\
\text { Index }\end{array}$ & 0.597 & 0.117 & 0.384 to 0.786 & $\begin{array}{c}81.8 \\
(48.2-97.2)\end{array}$ & $\begin{array}{c}57.1 \\
(28.9-82.2) \\
\end{array}$ & 1.91 & 1.91 \\
\hline AST/ALT & 0.601 & 0.115 & 0.387 to 0.789 & $\begin{array}{c}72.7 \\
(50.6-94.8)\end{array}$ & $\begin{array}{c}42.9 \\
(27.3-58.5)\end{array}$ & 1.31 & 1.25 \\
\hline
\end{tabular}

The areas under the ROC curves $(95 \% \mathrm{CI})$ were $0.627(0.412$ to 0.810$)$ for TyG index, $0.597(0.384$ to 0.786$)$ for TyG BMI index and 0.601 (0.387 to 0.789) for AST/ALT.

The ROC analysis in Table 2 shows that the TyG index has a better performance in comparison with the TyG BMI and AST/ALT. These findings are consistent with the study by E.K. Hameed et al. ${ }^{(18)}$
The ROC Curve analysis showed the optimal cut off value in cases was 4.55 for TyG index and 105 for TyG -BMI index.

The average value of TyG index was 4.55 and the average value of TyG-BMI index was 116.75 .

The positive linear regression was 2.55 for TyG index 1.91 and for TyG-BMI index indicating that test was associated with disease. Table 3

Table 3: Correlation between Studied Parameters

\begin{tabular}{|c|c|c|c|c|c|c|c|c|}
\hline & \multicolumn{4}{|c|}{ Cases } & \multicolumn{4}{|c|}{ Controls } \\
\hline & \multicolumn{2}{|c|}{ TyG Index } & \multicolumn{2}{|c|}{ TYG_BMI } & \multicolumn{2}{|c|}{ TyG Index } & \multicolumn{2}{|c|}{ TYG_BMI } \\
\hline & $\begin{array}{c}\text { Correlation } \\
\text { Coefficient }\end{array}$ & p-value & $\begin{array}{c}\text { Correlation } \\
\text { Coefficient }\end{array}$ & p-value & $\begin{array}{c}\text { Correlation } \\
\text { Coefficient }\end{array}$ & p-value & $\begin{array}{c}\text { Correlation } \\
\text { Coefficient }\end{array}$ & p-value \\
\hline Age (yrs) & 0.518 & 0.102 & 0.164 & 0.631 & -0.165 & 0.589 & -0.022 & 0.943 \\
\hline BMI & -0.127 & 0.709 & 0.809 & 0.003* & 0.000 & 1.000 & 0.771 & 0.001* \\
\hline $\begin{array}{c}\text { Fasting } \\
\text { TG }\end{array}$ & 0.980 & 0.001* & 0.282 & 0.400 & 0.827 & 0.001* & 0.459 & 0.098 \\
\hline $\begin{array}{c}\text { Fasting } \\
\text { BGL }\end{array}$ & 0.372 & 0.259 & 0.11 & 0.747 & 0.542 & $\mathrm{0.045}^{*}$ & 0.066 & 0.822 \\
\hline AST/ALT & -0.187 & 0.582 & 0.073 & 0.831 & 0.466 & 0.093 & 0.275 & 0.342 \\
\hline
\end{tabular}

In our study we found that in cases and controls the fasting TG was correlated with the TyG index and $p$ value was less than 0.05 , proving the statistical significance.

Fasting BGL was correlated with TyG index, significantly in controls.

In our study we found that the liver transaminase levels (AST and ALT) were normal. The average ratio of AST/ALT was 1.23 in cases and 1.0 in controls which was not correlated with TyG index and TyG BMI index.

\section{DISCUSSION}

Published studies regarding the association between PCOS and NAFLD are still very scarce and they evaluate populations with lifestyle and genetic backgrounds. ${ }^{(19)}$.

The prevalence of NAFLD in women with PCOS was found to be $35-70 \%$ in some studies while NAFLD in these patients presented in a more severe form. (10)

How one condition contributes to the other is the topic under research. The evidence indicates that NAFLD may exacerbate insulin resistance. Multiple proinflammatory, coagulant, and fibrogenic mediators are released, that may contribute to the pathophysiology of PCOS. On the other hand, insulin resistance and androgen excess are the main characteristics of PCOS that could exacerbate the risk of developing NAFLD. ${ }^{(20)}$

Liver has an important regulatory function in various metabolic processes. The hyperandrogenemia, dyslipidemia, hyperglycemia, IR and low-grade inflammation, all of which characterize PCOS, are concomitant factors that generate and aggravate nonalcoholic fatty liver disease (NAFLD) ${ }^{(21)}$ 
The post-receptor defect in insulin signal transduction pathways in glucose and lipid metabolism is the postulated cause for insulin resistance in PCOS ${ }^{(22)}$. As a result of selective insulin resistance, tissues other than skeletal muscles which express insulin receptors like the ovaries are also sensitive to insulin and are exposed to increased levels of circulating insulin. This hyperinsulinemia decreases the production of sex-hormone binding globulin (SHBG) from the liver. SHBG is the main protein which binds testosterone and prolongs its metabolic clearance. Thus there is increased testosterone bioavailability in these patients. Therefore, insulin resistance, which is a prominent finding in PCOS patients, is an important factor that contributes to ovarian androgen excess which is the hallmark of PCOS.

Thus we see that insulin resistance is a common link \& between NAFLD and PCOS, through a common pathophysiologic mechanism. Figure 6

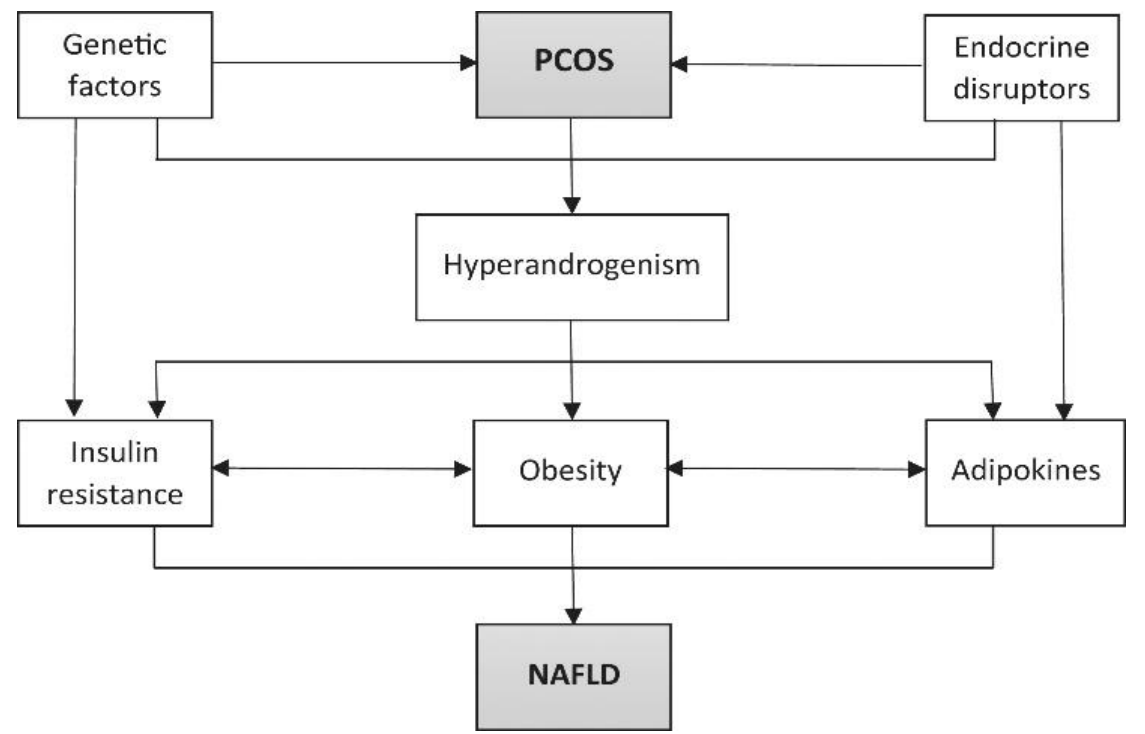

Fig.6 : Factors involved in the development of nonalcoholic fatty liver disease (NAFLD) in patients with polycystic ovary syndrome (PCOS).

The gold standard for the diagnosis of NAFLD is liver biopsy, but this method presents obvious limitations such as risk of complications due to invasive nature, sampling variability (heterogeneous parenchymal involvement) \& cost.

Liver function tests (serum samples) may show up within normal limits or have a fluctuating course in patients with NAFLD. In fact, severe forms of NAFLD have been described in patients with normal enzymes.

So, the TyG index and TyG-BMI index were used as markers of insulin resistance to predict the early NAFLD in women with PCOS.

The San Antonio Metabolism (SAM) study found that TyG index is significantly correlated with both hepatic insulin resistance and hepatic triglyceride content ${ }^{(24)}$.
Bongyoung Kim et al ${ }^{(25)}$ found that with sensitivity 62.1 and specificity 63.1 , the cut off value of TyG index was 4.69, results consistent with our study findings.

Cutoff value of TyG index for IR was estimated in few studies. In our study the ROC curve analysis showed the optimal cut off value for TyG index was 4.55 and for TyG-BMI index in PCOS as 104.76.

Er, L. K. et al, Zheng, S. et al., Lim, J., et al studies ${ }^{(11,26,27)}$ have found the cutoff value of TyG index as 4.66 in men and 4.57 in women. Fernando Guerrero-Romero et al. (28) found the best value of TyG index as 4.68 suggesting the utility of TyG index as marker of insulin resistance.

We have found TyG index as the better predictor of insulin resistance in PCOS women than TyG-BMI index indicating that the compound indices does 
not add the predictive value to the TyG index. This may be due to the smaller sample size in our study.

In our study we have found the significant correlation between the TyG index and fasting triglycerides and glucose levels in both cases and controls indicating the role of glucolipotoxicity in disease etiology.

So we suggest use of TyG index as a surrogate marker of insulin resistance.

It can be used as screening tool for early detection and identification of women with insulin resistance

Aminotransferase levels are one of the screening tools to evaluate the presence of NAFLD in patients with PCOS. However, aminotransferase levels may be normal in a large subset of patients who have NAFLD and not steatohepatitis.

Walter Schwimmer, Omid Khorran et al ${ }^{(29)}$ have shown that when PCOS patients were compared to age matched healthy women, the ALT levels were significantly higher in women with PCOS which contrasts our study findings.

In our study we found that the liver transaminase levels (AST and ALT) were normal. It was also not correlated with TyG index and TyG BMI index, indicating that using only transaminases as a marker of NAFLD in PCOS patients is of limited value. It also needs consideration that NAFLD being a progressive disorder with fluctuating course, addition of another modality like abdominal ultrasound may be helpful.

As per the available data, the degree of insulin resistance is directly proportional to the prevalence of both PCOS and NAFLD. NAFLD has already been proposed to be the hepatic manifestation of metabolic syndrome and PCOS as the ovarian manifestation of metabolic syndrome ${ }^{(11) .}$

It was recommended that liver evaluation by assessment of aminotransferase levels and abdominal ultrasound in PCOS patients that are obese or have features of metabolic syndrome can be considered as an initial screening tool for detecting NAFLD. ${ }^{(30)}$

The gynecologist especially infertility consultants have a paramount role in management of reproductive aspect of PCOS. They need to counsel the patients about the long-term health consequences of PCOS like metabolic syndrome \& NAFLD, emphasizing appropriate screening \& follow-up ${ }^{(31)}$.

\section{CONCLUSION}

1. TyG index could be an accessible and reliable test for estimating insulin resistance in low-income individuals in high-risk groups like PCOS in INDIA.

2. This index can be used for early identification of the cohort of women with PCOS with insulin resistance.

3. TG and glucose based mathematical markers like TyG index could be a good counseling tool for therapeutic lifestyle management in women with PCOS.

4. Using these parameters is less costly, measurements of glucose and triglycerides are available in all clinical laboratories. Insulin measurement is not required which is an expensive and less accessible test.

\section{Limitations of the study:}

1. TyG index and TyG-BMI index is not an established measure of IR.

2. TyG index and TyG-BMI index accuracy needs to be checked in patients with hypertriglyceridemia.

3. We could not get sufficient samples as patients were not particularly enthusiastic to take the tests to screen for long term consequences of PCOD, rather wanting to focus on eminent problem at hand, i.e. treatment of infertility or correction of menstrual irregularities. Some stated financial constraints.

4. The results should be viewed in light of a small sample size and limited duration. Larger sample size \& prolonged study duration will be helpful to study the interlink between TyG Index, TyG BMI 
Index \& serum transaminases in women with PCOS, ultimately reflecting upon the link between PCOS and NAFLD.

5. We propose screening of patients with PCOS with high values of TyG index with abdominal ultrasound \& liver transaminases for early detection of NAFLD.

\section{Acknowledgement: None}

Conflict of Interest: The authors declare that there is no conflict of interest.

\section{Source of Funding: None}

\section{Ethical Approval: Approved}

\section{REFERENCES}

1. KrishnamoorthyY, RajaaS, MuraliS, RehmanT, SahooJ, Kar SS (2020). Prevalence of metabolic syndrome among adult population in India: A systematic review and meta-analysis. PLoSONE 15(10):e0240971.

2. Huang PL. A comprehensive definition for metabolic syndrome. Dis Model Mech. 2009 May-Jun; 2(5-6):231-7.

3. Norman RJ, Dewailly D, Legro RS, Hickey TE. Polycystic ovary syndrome. Lancet. 2007 Aug 25; 370(9588):685-97.

4. Ganie MA, Kalra S. Polycystic ovary syndrome - A metabolic malady, the mother of all lifestyle disorders in women - Can Indian health budget tackle it in future? Indian J Endocrinol Metab. 2011; 15(4): 239-241.

5. Azziz R, Carmina E, Chen $Z$, et al. Polycystic ovary syndrome. Nat Rev Dis Primers 2016; 2:16057.

6. .Polyzos SA, Kountouras J, Zavos C. Nonalcoholic fatty liver disease: the pathogenetic roles of insulin resistance and adipocytokines. Curr Mol Med. 2009 Apr; 9(3):299-314.

7. Targher G. Non-alcoholic fatty liver disease, the metabolic syndrome and the risk of cardiovascular disease: the plot thickens. Diabet Med. 2007 Jan; 24(1):1-6.

8. Sanyal D, Mukherjee P, Raychaudhuri M, Ghosh S, Mukherjee S, Chowdhury S. Profile of liver enzymes in non-alcoholic fatty liver disease in patients with impaired glucose tolerance and newly detected untreated type 2 diabetes. Indian $\mathbf{J}$ Endocrinol Metab. 2015; 19(5):597-601.

9. .D. M. Torres and S. A. Harrison, "Diagnosis and therapy of nonalcoholic steatohepatitis," Gastroenterology, vol. 134, no. 6, pp. 1682-1698, 2008.

10. Sejti TL, Holland ND, Sanders LL, Pereira KC, Diehl AM, Brown AJ. Nonalcoholic steatohepatitis and nonalcoholic fatty liver disease in young women with polycystic ovary syndrome. J Clin Endocrinol Metb. 2006;91(5):1741-47.

11. Er, L. K. et al. Triglyceride glucose-body mass index is a simple and clinically useful surrogate marker for insulin resistance in nondiabetic individuals. PLoS ONE 11, e0149731 (2016).

12. Makbul Amana, Dewi Resnawitab, Haerani Rasyidc, The concordance of triglyceride glucose index (TyG index) and homeostatic model assessment for insulin resistance (Homa-IR) in non-diabetic subjects of adult Indonesian males Clinical Epidemiology and Global Health 9 (2021) 227-230

13. Zhang $\mathrm{S}, \mathrm{Du} \mathrm{T}$, Zhang $\mathrm{J}$, et al. The triglyceride and glucose index (TyG) is an effective biomarker to identify nonalcoholic fatty liver disease. Lipids Health Dis. 2017;16(1):15

14. Rotterdam ESHRE/ASRM-Sponsored PCOS consensus workshop group (2004). "Revised 2003 consensus on diagnostic criteria and long-term health risks related to polycystic ovary syndrome (PCOS)". Hum. Reprod. 19 (1): 41-7.

15. Jianhai Zhang*, Jian hu*, Chunxia Zhang, Yanni Jiao, Xiang Kong and Wei Wang, Analyses of risk factors for polycystic ovary syndrome complicated with non-alcoholic fatty liver disease, Experimental and Therapeutic Medicine 15: 4259-4264, 2018.

16. Available

"https://www.euro.who.int/en/healthtopics/disease-prevention/nutrition/ahealthy-lifestyle/body-mass-index-bmi.", as on on 21.4.2021.

17. R. Vidya Bharathi et al. Middle East Fertility Society Journal 22 (2017) 313-316

18. E.K. Hameed, Diabetes \& Metabolic Syndrome: Clinical Research \& Reviews 13 (2019) 560e563.

19. Cerda C, Pérez-Ayuso RM, Riquelme A, Soza A, Villaseca P, Sir-Petermann T, et al. Nonalcoholic fatty liver disease in women 
with polycystic ovary syndrome. J Hepatol. 2007; 47(3):412-7.

20. Targher G, Rossini M, Lonardo A. Evidence that non-alcoholic fatty liver disease and polycystic ovary syndrome are associated by necessity rather than chance: a novel hepato-ovarian axis? Endocrine. 2016; 51:211-221.

21. Burra P. Liver abnormalities and endocrine diseases. Best Practice and Research: Clinical Gastroenterology 201327 553-563.

22. Diamanti-Kandarakis E, Dunaif A. Insulin resistance and the polycystic ovary syndrome revisited: an update on mechanisms and implications. Endocr Rev. 2012 Dec; 33(6):981-1030.

23. Djuro Macut, Ivana Božić-Antić, Jelica Bjekić-Macut, Konstantinos Tziomalos, management of endocrine disease Polycystic ovary syndrome and nonalcoholic fatty liver disease, review, European Society of Endocrinology, 177:3 R145-R158

24. Guerrero-Romero, F. et al. The product of triglycerides and glucose, a simple measure of insulin sensitivity. Comparison with the euglycemic-hyperinsulinemic clamp. J. Clin. Endocrinol. Metab. 95, 3347-3351 (2010).

25. Kim B, Choi HY, Kim W, Ahn C, Lee J, Kim JG, Kim J, Shin H, Yu JM, Moon S. The cut-off values of surrogate measures for insulin resistance in the Korean population according to the Korean Genome and Epidemiology Study (KOGES). PLoS One. 2018 Nov 12; 13(11): e0206994.

26. Zheng, S. et al. Triglyceride glucose-waist circumference, a novel and effective predictor of diabetes in first-degree relatives of type 2 diabetes patients: Cross-sectional and prospective cohort study. J. Transl. Med. 14, 260 (2016).
27. Lim, J., Kim, J., Koo, S. H. \& Kwon, G. C. Comparison of triglyceride glucose index, and related parameters to predict insulin resistance in Korean adults: An analysis of the 2007-2010 Korean National Health and nutrition examination survey. PLoS ONE 14, e0212963 (2019).

28. Fernando Guerrero-Romero, Luis E. Simental-Mendía, Manuel González-Ortiz, Esperanza Martínez-Abundis, María G. Ramos-Zavala, Sandra O. HernándezGonzález, Omar Jacques-Camarena, Martha Rodríguez-Morán, The Product of Triglycerides and Glucose, a Simple Measure of Insulin Sensitivity. Comparison with the Euglycemic-Hyperinsulinemic Clamp, The Journal of Clinical Endocrinology \& Metabolism, Volume 95, Issue 7, 1 July 2010, Pages 33473351, https://doi.org/10.1210/jc.2010-0288

29. Walter Schwimmer, Omid Khorran et al.Available at, Women with PCOS at Increased Risk for Liver Disease (ucsd.edu)Accessed on 21.4.2021

30. Vassilatou E. Nonalcoholic fatty liver disease and polycystic ovary syndrome. World J Gastroenterol. 2014; 20(26):8351-8363.

31. Romanowski MD, Parolin MB, Freitas ACT, Piazza MJ, Basso J, Urbanetz AA. Prevalence of non-alcoholic fatty liver disease in women with polycystic ovary syndrome and its correlation with metabolic syndrome

How to cite this article: Gawade GC, Wadadekar GS, Padwal MK et.al. Correlation between TyG (fasting triglyceride-glucose) index, TyG BMI index \& liver transaminases in women with polycystic ovary disease syndrome. International Journal of Research and Review. 2021; 8(5): 193-201. DOI: https://doi.org/10. 52403/ijrr.20210527 